\title{
An L-band ultrasonic probe using polymer optical fibre
}

\author{
Broadway, Christian; Woyessa, Getinet; Bang, Ole; Mégret, Patrice; Caucheteur, Christophe
}

\section{Published in:}

Proceedings of SPIE

Link to article, DOI:

$10.1117 / 12.2510017$

Publication date:

2019

Document Version

Publisher's PDF, also known as Version of record

Link back to DTU Orbit

\section{Citation (APA):}

Broadway, C., Woyessa, G., Bang, O., Mégret, P., \& Caucheteur, C. (2019). An L-band ultrasonic probe using polymer optical fibre. In A. A. Oraevsky, \& L. V. Wang (Eds.), Proceedings of SPIE (Vol. 10878). [108780N] SPIE - International Society for Optical Engineering. Progress in Biomedical Optics and Imaging - Proceedings of SPIE https://doi.org/10.1117/12.2510017

\section{General rights}

Copyright and moral rights for the publications made accessible in the public portal are retained by the authors and/or other copyright owners and it is a condition of accessing publications that users recognise and abide by the legal requirements associated with these rights.

- Users may download and print one copy of any publication from the public portal for the purpose of private study or research.

- You may not further distribute the material or use it for any profit-making activity or commercial gain

- You may freely distribute the URL identifying the publication in the public portal 


\section{An L-band ultrasonic probe using polymer optical fibre}

Christian Broadway, Getinet Woyessa, Ole Bang, Patrice Mégret, Christophe Caucheteur

Christian Broadway, Getinet Woyessa, Ole Bang, Patrice Mégret, Christophe Caucheteur, "An L-band ultrasonic probe using polymer optical fibre," Proc. SPIE 10878, Photons Plus Ultrasound: Imaging and Sensing 2019, 108780N (27 February 2019); doi: 10.1117/12.2510017

SPIE. Event: SPIE BiOS, 2019, San Francisco, California, United States 


\title{
An L-band ultrasonic probe using polymer optical fibre Christian Broadway ${ }^{l}$, Getinet Woyessa ${ }^{2}$, Ole Bang ${ }^{2}$, Patrice Mégret $^{l}$, Christophe Caucheteur ${ }^{l}$ ${ }^{l}$ Electromagnetism and Telecommunication Department, University of Mons \\ ${ }^{2}$ DTU Fotonik, Denmark Technical University
}

\begin{abstract}
In recent years, Polymer optical fibre (POF) has been receiving increasing attention for sensing applications. For applications such as endoscopic ultrasound and photo-acoustics, PMMA polymer fibres deliver ever improving performance and more types of polymer are being trialled for these applications. The fundamental properties of POF, when correctly leveraged, deliver at least an order of magnitude in improvements over silica fibres. POF delivers lower acoustic impedance, a reduced Young's Modulus and a higher acoustic sensitivity within the megahertz region. In contrast, existing piezo-electric transducers have an inherent narrow acoustic bandwidth and a proportionality to size that causes difficulties for applications such as endoscopy within the biomedical domain.

With the increasing take-up of POF, improvements have been made in fibre dopant distribution, the range of polymers available and connectorisation techniques. While newer polymer fibres are under preliminary study, PMMA has shown itself to be the most sensitive fibre to date despite historically higher levels of dopant inconsistency and an implementation around $830 \mathrm{~nm}$ that has a lower signal-to-noise ratio than possible. The prior approach of edge filtering a Bragg grating with a high speed photodiode has been shown to function and is therefore maintained.

We present a step index PMMA Bragg grating ultrasonic probe in the L-band for the first time. Detection is achieved using a Bragg grating less than $1 \mathrm{~cm}$ in length in a fibre under $10 \mathrm{~cm}$ long. We examine the temporal and frequency response of the sensor over a $1-15 \mathrm{MHz}$ range.
\end{abstract}

Keywords: Polymer optical fibre, fibre sensors, biomedical applications, ultrasound detection, fibre Bragg gratings, PMMA

\section{INTRODUCTION}

Ultrasonic detection is widely spread over numerous diagnostic applications in the biomedical domain, especially when considering the increasing attention that is illuminating the field of photo-acoustic imaging (PAI). PAI has been reported for disciplines including cardiology and neurology [1] using techniques such as microscopy and endoscopy that on occasion consider multiple modality systems [2]. The wide range of applications, disciplines, techniques and modalities inevitably introduce a wide variety of application constraints that render numerous detectors favourable for some applications and unfavourable for others.

While historically, the baseline for comparison are piezo-electric transducers that rely on resonance effects, limiting frequency bandwidth and linking sensitivity to the size of the sensing element. Furthermore, they are sensitive to electromagnetic interference, a cause for concern for certain applications. For many years, researchers have proposed silica optical fibres as a step forward, offering immunity to such interference and providing small sensor sizes unfettered by resonance limitations. However, while silica fibre sensors are a common proposal in many fields of industry, their low intrinsic sensitivity to mechanical and thermal perturbations tends to lead to complex techniques in the quest for an ever more sensitive sensor.

An alternative to silica fibres are their polymer optical fibre (POF) counterparts, which have existed for a similar length of time but have been overlooked due to their larger developmental challenges at the time [3]. Nonetheless, in the last decade significant research has been invested into their improvement and implementation, leading to developments such as readily available effectively single mode POF, a wider variety of fibre materials and lower attenuation [4,5]. Today, polymer fibres are being investigated in multiple wavelength regions for a range of ever more complex applications seeking to take advantage of their increased sensitivity to mechanical and thermal effects [3].

Photons Plus Ultrasound: Imaging and Sensing 2019, edited by Alexander A. Oraevsky, Lihong V. Wang Proc. of SPIE Vol. 10878, 108780N · C 2019 SPIE · CCC code: 1605-7422/19/\$18 · doi: 10.1117/12.2510017 
Research into ultrasound detection using POF began approximately 10 years ago in an interferometric configuration using poly(methyl methacrylate) (PMMA) [6]. To be considered for a wider variety of applications, the transition to Fibre Bragg Gratings (FBGs) took place to make probe formats possible and reduce the sensor size down to 1 centimetre in length. This transition produced results showing its sensing capability in part in the $850 \mathrm{~nm}$ wavelength region [7]. However, while many improvements have been demonstrated, the wavelength region is uncommon and significant sensitivity improvements need demonstrating before POF can be considered competitive against silica fibre sensors of the day.

In the last year, the transition to the L-band of the wavelength spectrum has been demonstrated for ultrasound detection along with a new polymer (CYTOP) and sensor in the form of a tilted FBG [8]. This wavelength transition enabled a more interesting polymer and now allows a serious investigation to show which polymer is best in fundamental terms for ultrasonic detection. Additionally, the use of some technology is more economic, such as fibre circulators, allowing system improvements at lower costs. While PMMA has extremely high attenuation in the L-Band, the now proven process of polymer direction connection into standard FC/APC connectors enables the use of much shorter lengths of fibre as the failure rate is uniquely dictated by success or failure of FBG inscription. Shorter lengths of PMMA arguably mitigates the higher attenuation, rendering it still attractive for ultrasonic detection. Finally, femtosecond inscription techniques have been demonstrated for inscribing FBGs within PMMA in the L-band [9], leading to higher reflected spectral profiles.

This paper builds on our initial work in the L-band by presenting a step index PMMA FBG sensor less than $1 \mathrm{~cm}$ long in a fibre shorter than $10 \mathrm{~cm}$ in length. The FBF is inscribed and connectorised, then exposed to incident ultrasound over a 1 $-15 \mathrm{MHz}$ range.

\section{METHODOLOGY AND EXPERIMENTAL SETUP}

To detect ultrasound, we tune a laser to the $3 \mathrm{~dB}$ point of the FBG and use it as an edge filter. This technique tracks changes in the amplitude of the laser that are caused spectral profile shifts from the applied pressure of the incident ultrasonic wave. These changes trace out the incident signal where the form of the output wave is dependent on the bandwidth of the FBG relative to the sensitivity of the fibre to pressure. We resume this detection method in Fig. 1.

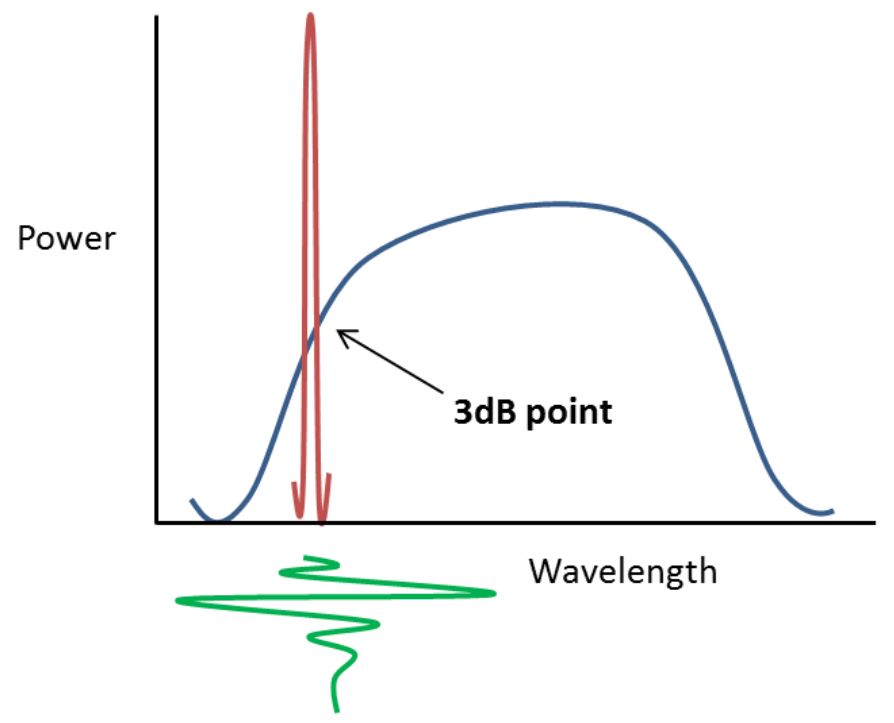

Figure 1 - The spectral profile of the FBG (blue) is stabilised and the $3 \mathrm{~dB}$ point identified on one of the slopes. The tuneable laser (red) is tuned to this point. Incident ultrasonic waves cause a shift in the wavelength and an oscilloscope output (green) representing the initial wave. 
Our sensor is inscribed in step index PMMA created by the Hong Kong Polytechnic University with a core diameter of $8.2 \mu \mathrm{m}$ and a cladding diameter of $150 \mu \mathrm{m}[9,10]$. The core and cladding are pure PMMA, with the core doped with diphenyl sulfide (5\% mole) and trans-4-stilbenemethanol (1\% w.t.). We inscribe the fibre with a He-cd laser using the procedure outlined in [11], delivering a spectral peak power significantly higher than previously used for ultrasonic detection. We connect the FBG to silica fibre using the UV gel curing method and examine the reflected spectral profile using a micron optics interrogator (NI PXIe-4844), shown in Fig. 2.

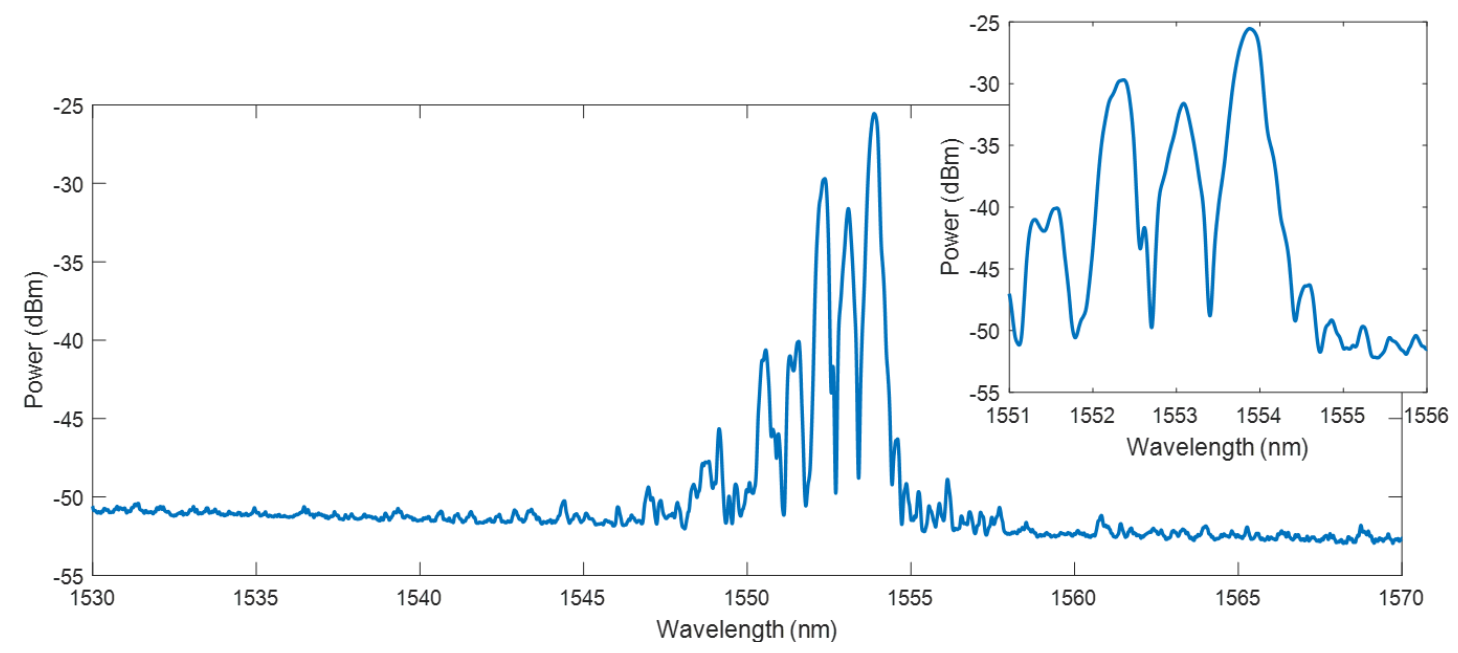

Figure 2 - FBG reflected spectral profile.

The FBG in question is few-mode, reflecting the multi-mode nature of step index PMMA Three primary peaks are observed with an approximate $1 \mathrm{~nm}$ spacing and a maximum signal-to-noise power of approximately $25 \mathrm{~dB}$ for the strongest peak at $1554 \mathrm{~nm}$. This peak is selected for having the highest power and the narrowest shape. To prepare the FBG for ultrasonic detection, we mount the fibre on a custom made support and immerse it in water, leaving it for 12 hours to stabilise, as PMMA is inherently humidity sensitive and has a rapid and considerable water ontake when immersed. Once stable, we tune our tuneable laser (Agilent 81940A) to the $3 \mathrm{~dB}$ point of the reflected spectrum and detect the variations in optical power using a photodiode (Menlo FPD 510). Our setup is depicted as Fig. 3.

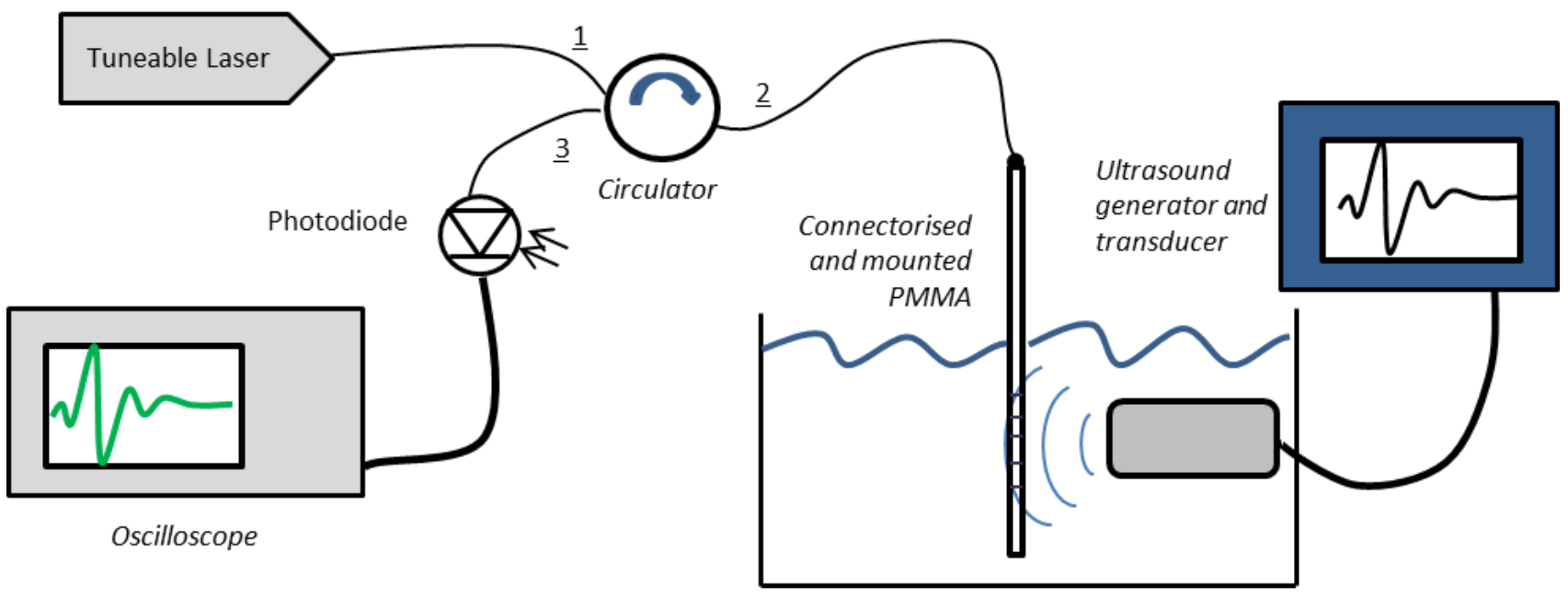

Figure 3 - Ultrasonic detection optical setup. Incident planar ultrasound is generated by a piezo-electric transducer with water based acoustic coupling and detected by optical means through the use of edge filtering. 
To generate ultrasound over the specified range, we use 4 Olympus unfocussed immersion transducers emitting ultrasound at 1 (V303-SU), 5 (V309-SU), 10 (V311-SU) and $15 \mathrm{MHz}$ (V319-SU) along with the associated base unit (EPOCH-650) to provide the excitation voltage.

\section{ULTRASONIC RESULTS AND ANALYSIS}

Using the aforementioned POF sensor, we detect incident ultrasound at 1, 5, 10 and $15 \mathrm{MHz}$ and analyse the received signals. For this experiment, each transducer is placed $25 \mathrm{~mm}$ away from our detector in turn. We ensure good alignment by optimising rotation, $\mathrm{X}$ and $\mathrm{Y}$ positioning as a calibration step using the $10 \mathrm{MHz}$ transducer. All detected signals are without averaging and using an oscilloscope with a $100 \mathrm{MHz}$ bandwidth. Fig. 4 shows the detected $1 \mathrm{MHz}$ ultrasound.
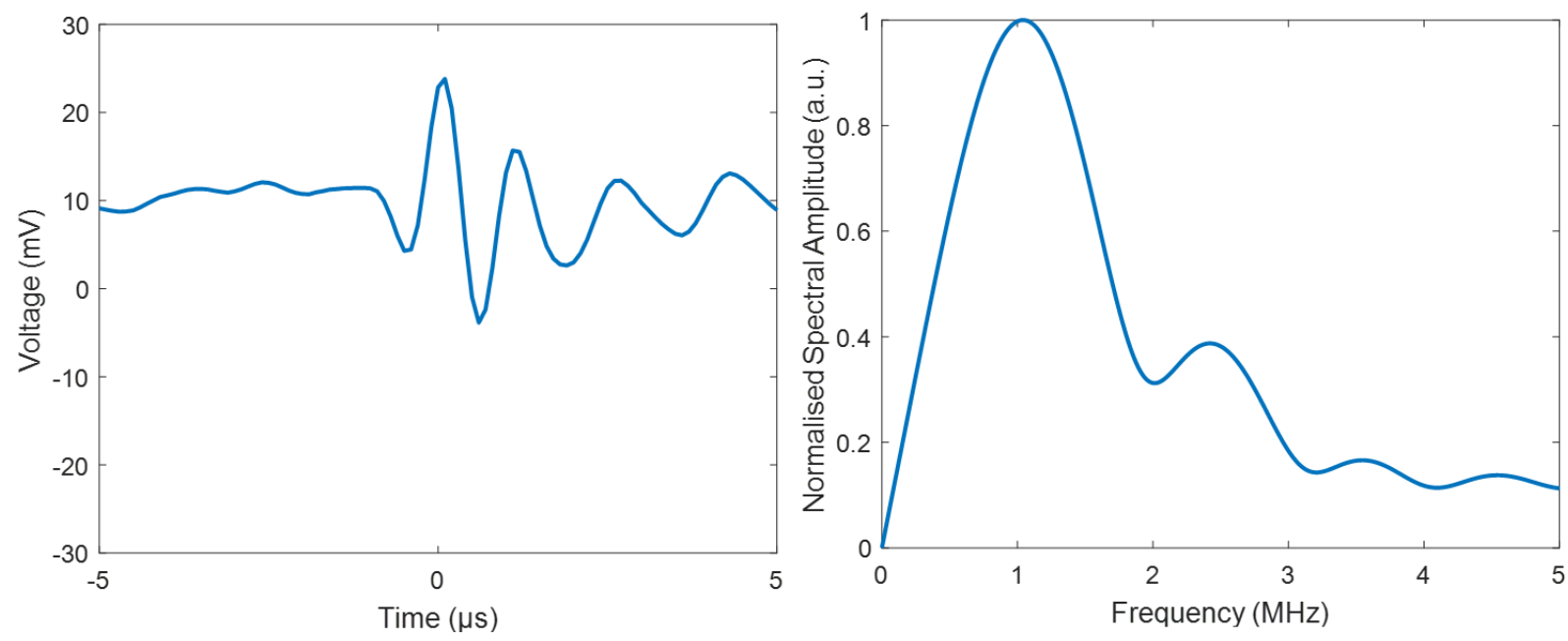

Figure 4-1 MHz ultrasound detected using our POF ultrasonic sensor with a transducer excitation voltage of $400 \mathrm{~V}$.

Our detector produces a $30 \mathrm{mV}$ peak-to-peak signal, the first two peaks clearly observable and the following two extremely close to the effective noise level of the system. An electrical filter is applied to limit the detected frequency to DC - $1 \mathrm{MHz}$ in order to clearly distinguish our signal from a noise level of $4 \mathrm{mV}$ with a base voltage varying over time in a $30 \mathrm{mV}$ range. We estimate an effective noise level of $4 \mathrm{mV}$ and define the peak-to-peak SNR as the ratio between the peak-to-peak signal and the effective noise level of the system, giving a peak-to peak SNR of 7.5. The detection of 1 $\mathrm{MHz}$ ultrasound is poor given the low SNR. While the acoustic filter was necessary to deliver a good result, the filter eliminates $50 \%$ of the power at $1 \mathrm{MHz}$ and even more beyond it. Furthermore, it is not yet possible to observe $1 \mathrm{MHz}$ ultrasound at lower excitation voltages.

We can observe from the frequency response that the transducer has a significant frequency component in this region and as a result, the amplitude of the signal would likely be much higher with a more appropriate acoustic filter, such as DC $0.5 \mathrm{kHz}$. Nonetheless, our frequency response corresponds well with the calibration test of the transducer where the peak was $0.87 \mathrm{MHz}$ and the $3 \mathrm{~dB}$ points were 0.64 and $1.19 \mathrm{MHz}$ respectively. Our wider $3 \mathrm{~dB}$ points of $0.4 \mathrm{and} 1.7 \mathrm{MHz}$ may simply be down to our sensor being naturally more wideband than the detecting transducer among other potential causes. The peak response being at $1.03 \mathrm{MHz}$ is a slight deviation, this may be caused by some angular misalignment and merits further investigation. Despite these less than ideal results, the overall performance of our detector is better than reported in the $850 \mathrm{~nm}$ region at maximum excitation voltages while worse at lower excitation levels.

Fig. 5 shows the detected $5 \mathrm{MHz}$ ultrasound signal, where a significantly improved performance relative to $1 \mathrm{MHz}$ detection is apparent. The signal amplitude is now an order of magnitude higher, delivering a $470 \mathrm{mV}$ peak-to-peak 
amplitude for the same maximum excitation voltage. The effective noise level remains $4 \mathrm{mV}$, yielding a peak-to-peak SNR of 117.5. These signals were obtained without acoustic filters. The temporal response of the system shows some deformation of the signal waveform depending on the excitation voltage. This can be due to a high incident pressure combined with non-linearity of the shape of the FBG spectral profile or saturation of the detection system among other explanations. Further studies can profitably determine the cause and the correction for these issues, which nonetheless preserve the frequency response and the general waveform. When compared to previous work, these results are significantly better when considering signal amplitude and SNR.
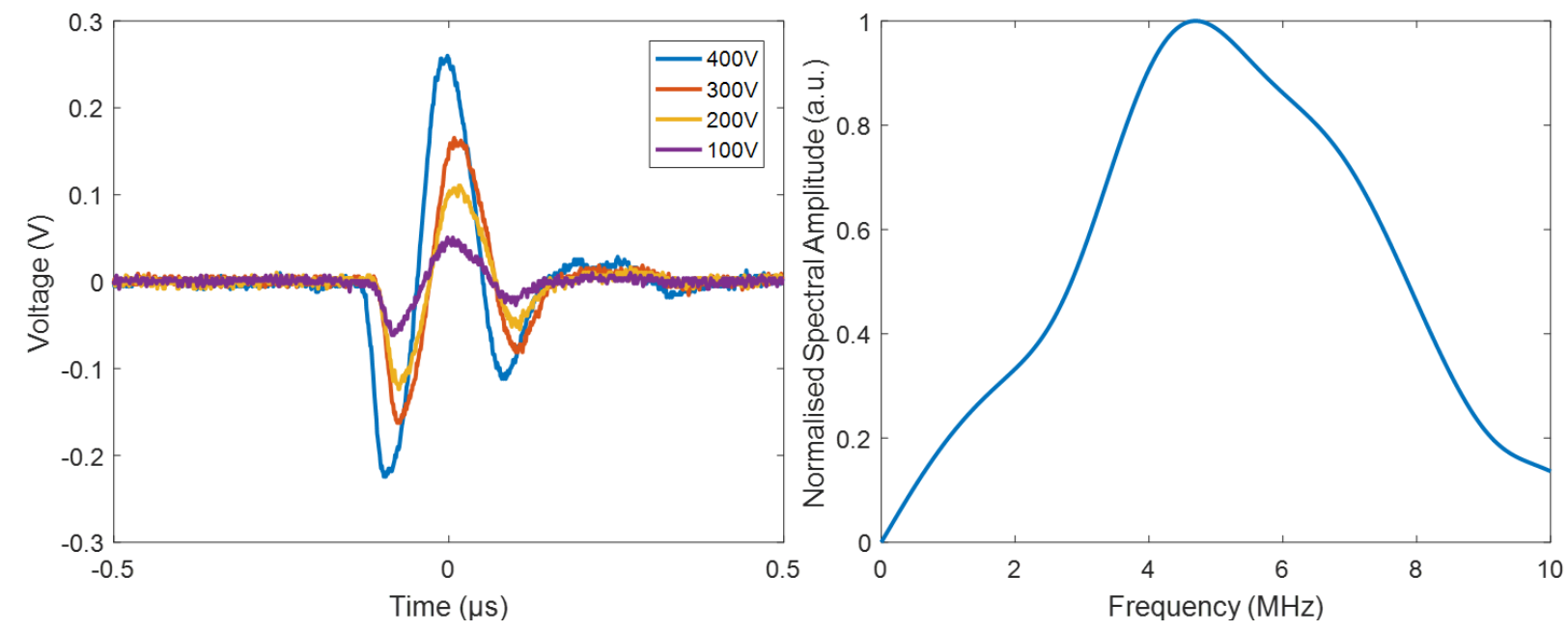

Figure $5-5 \mathrm{MHz}$ ultrasound detected using our POF ultrasonic sensor over a range of excitation voltages. The frequency response is taken using the maximum excitation voltage.

The frequency response of our sensor is in general harmony with that of the calibration test of our transducer; a peak value of $4.45 \mathrm{MHz}$ and $3 \mathrm{~dB}$ cut-off points at 3.16 and $6.45 \mathrm{MHz}$ respectively. Our sensor delivers a peak value of 4.7 $\mathrm{MHz}$ and $3 \mathrm{~dB}$ cut-off points at 2.8 and $7.85 \mathrm{MHz}$. As with the $1 \mathrm{MHz}$ frequency response, we see a general harmony in results, with a wider bandwidth than in the calibration test and a slightly higher frequency peak.
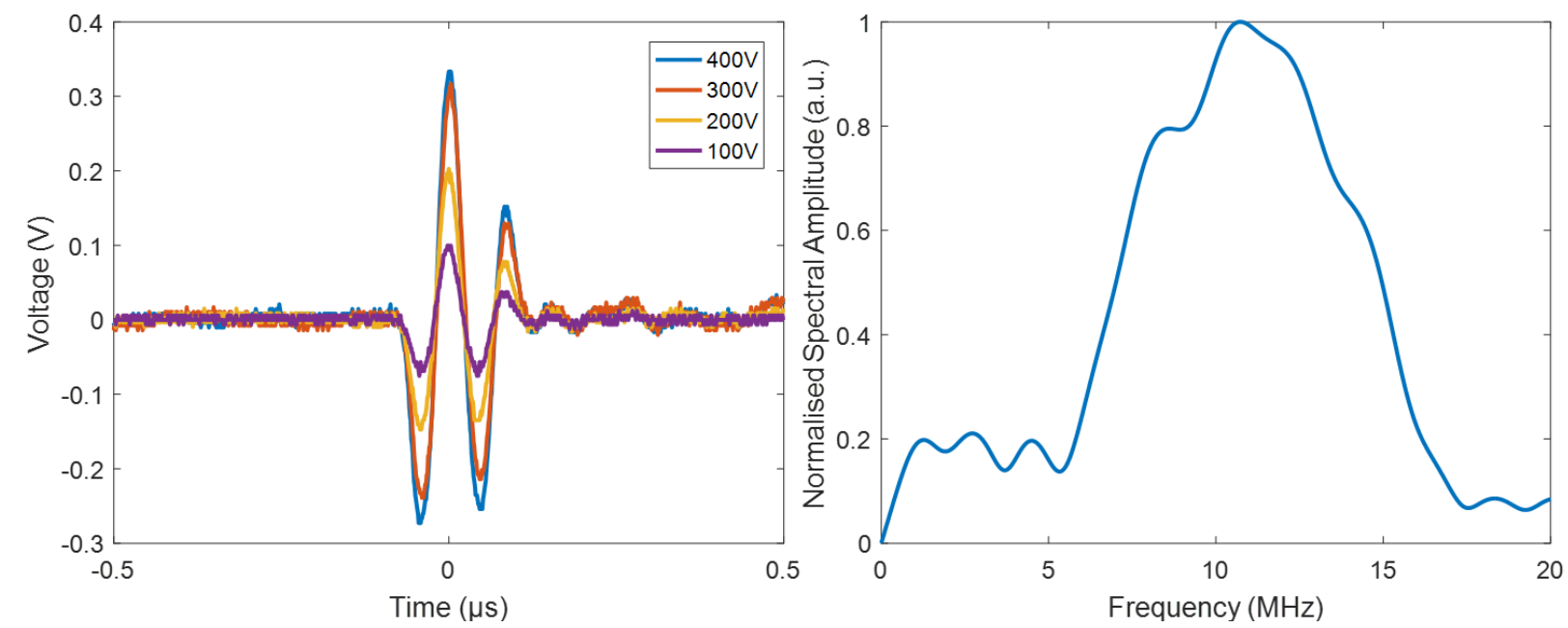

Figure 6-10 MHz ultrasound detected using our POF ultrasonic sensor over a range of excitation voltages. The frequency response is taken using the maximum excitation voltage.

Fig. 6 shows that the $10 \mathrm{MHz}$ results deliver even better results than those previously analysed, with no acoustic filtering. The peak-to-peak voltage now exceeds $0.6 \mathrm{~V}$ with the same effective noise level as previously mentioned. This delivers a 
peak-to-peak SNR value of 150 . Unlike the $5 \mathrm{MHz}$ results, we observe no waveform alterations as the excitation voltage is increased, suggesting that the causes of the saturation effect previously observed are likely frequency limited. We observe less difference between the $300 \mathrm{~V}$ and $400 \mathrm{~V}$ excitation levels, suggesting some limitation that does not produce a linear increase in amplitude for this transition.

The frequency response in this instance is more irregular in terms of shape. The calibration test gives a peak value of $10.33 \mathrm{MHz}$ with cut-off points at 7.62 and $13.36 \mathrm{MHz}$ respectively. Our frequency response peaks at $10.71 \mathrm{MHz}$ and has $3 \mathrm{~dB}$ points at 7.019 and $14.95 \mathrm{MHz}$. As before, our bandwidth appears wider and our peak is similar distance away from the peak in the calibration test. Further studies will be useful to determine why the frequency response is not a smooth peak and why in particular it plateaus briefly in one place.
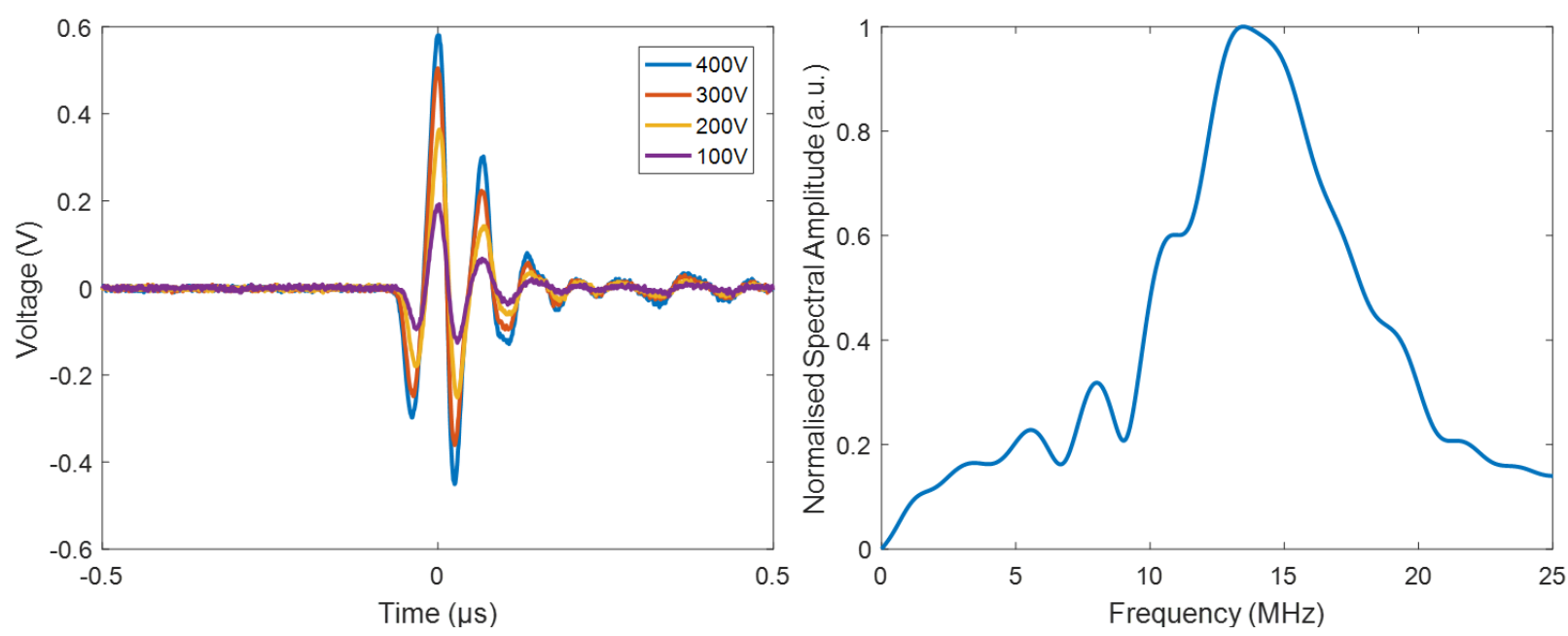

Figure $7-15 \mathrm{MHz}$ ultrasound detected using our POF ultrasonic sensor over a range of excitation voltages. The frequency response is taken using the maximum excitation voltage.

Finally, Fig. 7 shows the $15 \mathrm{MHz}$ frequency response with no acoustic filters. We observe a $1.03 \mathrm{~V}$ peak-to-peak ultrasonic response giving the peak-to-peak SNR as 257.5. As with the $10 \mathrm{MHz}$ response, the temporal response does not distort or change with increasing pressure and the 300-400 V transition exhibits the same smaller amplitude increase previously discussed. The frequency response peaks at $13.49 \mathrm{MHz}$ with cut-off points at 10.07 and $17.88 \mathrm{MHz}$. The calibration test for the emitter shows a peak at 15.66 and cut-off points at 11.6 and $19.7 \mathrm{MHz}$ respectively. This shows that our sensor has a smaller bandwidth than that of the calibration receiver and suggests an angular misalignment when considering the significantly different peak values between our sensor and the calibration test. One possible explanation is that the frequency bandwidth of the sensor may decrease depending on the angle of incidence to the sensor. This can occur for physical alignment reasons as well as if the FBG is not perfectly centred in the fibre core and will have an angular preference as a result. Further studies will be necessary to determine why these bandwidth discrepancies are observed.

We have exposed our sensor to a detailed analysis in the temporal and frequency domains. Our sensor shows a clear preference for the $5-15 \mathrm{MHz}$ region and delivers higher SNRs than previously reported. While some details point to unknown factors that would be profitable explored in future studies, we may conclude that the principle of detection over a $15 \mathrm{MHz}$ bandwidth has been demonstrated to an acceptable level of performance.

\section{CONCLUSION}

This paper has presented a step index PMMA FBG ultrasound sensor operating in the L-band of the optical spectrum. Our sensor is $7 \mathrm{~cm}$ long with an actual sensing length of less than $1 \mathrm{~cm}$ and has detected ultrasound over a $1-15 \mathrm{MHz}$ range. This configuration presents much higher signal to noise ratios than previous systems and provides a solid base on which to work. In particular, the improvements in the peak power of the FBG and the optimisation of the detection 
system have delivered strong results that can be further improved. In the future, our research will progress to direct connectorisation, consider a full characterisation of the sensor and compare polymers in a meaningful way to select a definitive polymer best optimised for ultrasonic detection in the present day.

\section{ACKNOWLEDGEMENTS}

C. Caucheteur is an Associate Researcher of the F.R.S.-FNRS and was recipient of an ERC (European Research Council) Starting Grant (Prosper). C. Broadway is supported by the Horizon 2020 project Modern2020.

\section{REFERENCES}

[1] Y. Zhou, J. Yao and L.V. Wang, "Tutorial on Photoacoustic Imaging", Journal of Biomedical Optics 21(6), 2016

[2] I. Olefir, E. Mercep, N. C. Burton, S. V. Ovsepian, V. Ntziachristos, "Hybrid multispectral optoacoustic and ultrasound tomography for morphological and physiological brain imaging", Journal of Biomedical Optics 21(8), 2016

[3] C. Broadway, M. Rui, A. G. Leal Junior, C. Marques and C. Caucheteur, "Towards commercial polymer fiber Bragg grating sensors: review and applications," Journal of Lightwave Technology, 2019

[4] G. Woyessa, A. Fasano, A. Stefani, C. Markos, K. Nielsen, H. K. Rasmussen, and O. Bang, "Single mode step-index polymer optical fiber for humidity insensitive high temperature fiber Bragg grating sensors," Opt. Express 24(2), 12531260 (2016).

[5] G. Woyessa, "Speciality and Microstructured Polymer Optical FBG Sensors", Ph.D. dissertation, DTU Fotonik, Department of Photonics Engineering, Technical University of Denmark, 2800 Kgs. Lyngby, Denmark, (2017).

[6] D. Gallego and H. Lamela, "High-sensitivity ultrasound interferometric single-mode polymer optical fibre sensors for biomedical applications," Optics Letters 34(12), 2009

[7] C. Broadway, D. Gallego, A. Pospori, M. Zubel, D. J. Webb, K. Sugden, G. Carpintero, and H. Lamela, "Microstructured polymer optical fiber sensors for opto-acoustic endoscopy," Proc. SPIE 9886, Micro-Structured and Specialty Optical Fibers IV, 98860S, 2016.

[8] C. Broadway, K. Kalli, A. Theodosiou, M. Zubel, K. Sugden, P. Mégret and C. Caucheteur, "L-band CYTOP Bragg gratings for ultrasound sensing," Proc. SPIE 10681, Micro-Structured and Specialty Optical Fibers V, no. 1068109, 2018.

[9] H. Y. Tam, Chi-Fung Jeff Pun, Guiyao Zhou, X. Cheng and M. L. V. Tse, Special structured polymer fibers for sensing applications, Optical Fibre Technology 16 pp. 357-366, 2010

[10] X. Hu, D. Kinet, K. Chah, C. Jeff Pun, H. Tam and C. Caucheteur, "Bragg grating inscription in PMMA optical fibers using 400-nm femtosecond pulses," Optics Letters (42)14, pp. 2794-2797, 2017

[11] X. Hu, D. Kinet, K. Chah, P. Mégret, and C. Caucheteur, "Bragg gratings inscription at $1550 \mathrm{~nm}$ in photosensitive step-index polymer optical fiber," Proc. SPIE 8794, Fifth European Workshop on Optical Fibre

Sensors, 87942Q, 2013 